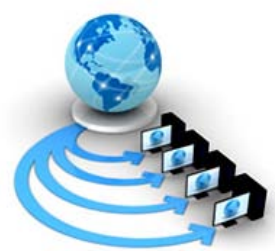

\title{
PERFORMANCE EVALUATION OF HYBRID SWARM INTELLIGENCE TECHNIQUES FOR EXTRACTION AND RECOGNITION OF FACIAL FEATURES
}

\author{
Ravneet Kaur (Author) \\ P.G. Student, CSE \\ DAVIET, Jalandhar \\ Punjab, India
}

\author{
Rajeev Kumar \\ Professor, Dept. of IT \\ DAVIET, Jalandhar \\ Punjab, India
}

\author{
Reeta Bhardwaj \\ Professor, Dept. of CSE \\ DAVIET, Jalandhar \\ Punjab, India
}

\begin{abstract}
The two most important steps in the face recognition procedure are Feature Extraction and Feature Selection. Metaheuristic approach is applied for recognition of a face in this work. First of all, the features are selected using the Discrete Cosine Transformation technique. On these selected features Particle Swarm Optimization technique is applied to select the meaningful features. In the end, Ant Colony Optimization approach is applied on those selected features to recognize the facial image. This is done by measuring distance between the features selected. The results are compared using the parameters Accuracy, Training Time, Recognition Time, Mean Square Error (MSE) and Peak Signal to Noise Ratio (PSNR). The results show the significant increase in the accuracy, PSNR and MSE values using hybrid approach than the Ant Colony Optimization and Particle Swarm Optimization techniques.
\end{abstract}

Keywords: Face Recognition, Hybridization, Feature Extraction, Feature Selection, Discrete Cosine Transformation (DCT), Ant Colony Optimization (ACO), Particle Swarm Optimization (PSO).

\section{INTRODUCTION}

A human face tells a great amount of information to another person. It tells about the mood, intentions, attentiveness as well as the identity of a person. A person can be recognized by other ways like voice, finger prints, body shape etc. when the detail of face is not available. Still face is the most widely used method to check and identify a person's identity.

During the past few years, face recognition has recently got a large amount of attention as it is one among the most successful image understanding and analysis application. This happens because of the following two reasons: first one is after years of research the development and large scale availability of technologies and second is its use in areas where security of a person's identity is a main challenge. So there was a strong need of some systems, which have to be user-friendly but as well as can protect and secure our privacy and assets without losing our identity.

The type of input given to this facial recognition system is to be a video stream or an image. The output obtained from this system is the verification or identification of the person or group of persons who are present in the selected video or an image. The face recognition system can be defined as a 3 step process.

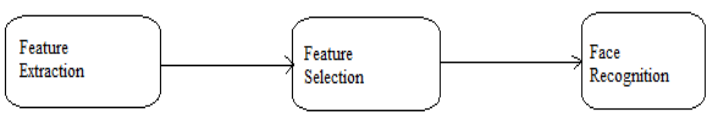

Fig 1 A 3 step process for face recognition

\section{Feature Extraction}

The "feature extraction" is considered as a preclassification step in most image classification systems [1]. To recognize a face of a person, some features are to be extracted from the selected image. Eyes, mouth, chin, nose, forehead, shape of the face etc. are considered as the special features. In most of the cases, the extracted features play an important role in the performance of face recognition systems because they need to be selected with great care and can affect the further work [1]. As the images of persons are usually changed because of their expressions or are rotated and scaled, it is advisable to define (or design) the features of image so that these features always remain unchanged because of the above said changes.

2. Feature Selection

The selected features are used to recognize the facial image of a person. So in the facial recognition process, feature selection has become its very important part. It is the process of finding only the meaningful features from the group of extracted features which form a specific pattern in the data set and helps in achieving our goal.

The face recognition work is done in three phases or steps. In first step Discrete Cosine Transformation is used to extract the features from an image. In the second step, Particle Swarm Optimization technique selects the meaningful features from the extracted features. Last and the third step consist of Ant Colony Optimization (ACO) algorithm which is based on the output of the Particle Swarm Optimization algorithm. 
The paper consists of five sections. Section 2 followed by the Introduction section gives the overview of the techniques used. The next section i.e. section 3 gives the detail description of the proposed algorithm. Section 4 explains the results and section 5 contains the conclusion of the paper followed by future scope of the proposed method.

\section{AN OVERVIEW OF USED TECHNOLOGIES}

\section{Discrete Cosine Transformation}

DCT has emerged as a popular transformation technique widely used in signal and image processing [2]. This is due to its strong "energy compaction" property where most of the signal information tends to be concentrated in a few low frequency components of the DCT [2]. This makes DCT a very useful tool for signal representation both in terms of information packing and in terms of computational complexity due to its data independent nature [3]. DCT separates the image into spectral sub-bands of different frequencies. These frequencies depend on the image's visual quality. The general equation for Discrete Cosine Transform of an image $f(x, y)$ of size $N \times M$ is defined by the following equation [2]:

$\mathrm{F}(\mathrm{u}, \mathrm{v})=\alpha(\mathrm{u}) \alpha(\mathrm{v}) \sum_{x=0}^{N-1-M-1} \cdot \cos \left[\frac{\pi \cdot u}{2 . N}(2 x+1)\right] \cos \left[\frac{\pi \cdot u}{2 . M}(2 y+1)\right] f(x, y)$

where $f(x, y)$ is the intensity of the pixel in row $x$ and column $y ; u=0,1, \ldots ., N-1$ and $v=0,1, \ldots ., M-1$ and the functions $a(u), a(v)$ are defined as [2]:

$$
\begin{array}{ll}
\alpha(\mathrm{u}) \alpha(\mathrm{v})=\left\{\sqrt{\frac{1}{N}}\right. & \text { for } \mathrm{u}, \mathrm{v}=0 \\
\alpha(\mathrm{u}) \alpha(\mathrm{v})=\left\{\sqrt{\frac{2}{N}}\right. & \text { for } \mathrm{u}, \mathrm{v} \neq 0
\end{array}
$$

\section{Swarm Intelligence Techniques}

Behaviour of variety of the interesting insect or animal in nature has always been captivating the researchers [4]. Swarm Intelligence is an innovative distributed intelligent paradigm for solving optimization problems inspired [4] from the biological examples by swarming, flocking and herding phenomena in vertebrates $[4,5]$. Swarm Optimization incorporates swarming behaviors observed in flocks of birds, schools of fish, swarms of bees, ant colonies and even human social behavior, from which the idea of nature inspired intelligence is emerged [4].

\section{i. Particle Swarm Optimization}

PSO algorithm was first described in 1995 by James Kennedy and Russell C. Eberhart [6]. It was inspired by the social behaviour of group of fish and group of birds. Just like other search based computational algorithms, Particle Swarm Optimization algorithm is also a population based searching algorithm. Recently PSO has been applied as an effective optimizer in many domains such as training artificial neural networks, linear constrained function optimization, wireless network optimization, and data clustering [2]. PSO is a population based stochastic optimization method which involves a number of particles flying around in the search space, where the position of each particle signifies a possible solution to the optimization problem [1].

\section{ii. Ant Colony Optimization}

ACO is a search method algorithm based on the behaviour of some ant species. Ants are the social creatures that work in groups to find food [4]. In ACO, proposed by Margo Dorigo [7], a number of artificial ants build solutions to optimization problems and exchange information on the quality of these solutions through a communication scheme that is evocative of the one adopted by real ants[4]. Ant colony optimization algorithm is iterative in nature. In each iteration, a number of ants travel from their nests in search of food [4]. Every ant constructs a solution by moving from one vertex to another vertex on the graph with one fixed rule i.e. not visiting any vertex that has already been visited in the path. At each step of the solution construction, an ant selects the following vertex to be visited according to a mechanism that is biased by the pheromone [4]. At the end of an iteration, on the basis of the quality of the solutions constructed by the ants, the pheromone values are modified in order to bias ants in future iterations to construct solutions similar to the best ones previously constructed [4].

\section{PROPOSED ALGORITHM}

After training the database, this proposed hybrid work is composed of two parts. The first part consists of Particle Swarm Optimization technique which is used to select the meaningful features from the group of features obtained through DCT. In the second part Ant Colohiy) Optimization algorithm is used to match the selected features with the images stored in the database. The figure 2 displays the steps followed by proposed work to recognize the facial image.

1. Selecting the Features Using PSO

Before feature selection using PSO, we should assume that each particle maintains:

i. $\quad$ Position in search space (x)

ii. Velocity

iii. Individual best position called pbest

iv. Swarm maintains its best position called gbest

Steps necessitate in selecting the features using PSO algorithm are:

Step- 1 (Initialization): Assign an initial value to all the particles of population having random velocity $\left(\mathrm{v}_{\mathrm{i}}\right)$ and location $\left(\mathrm{x}_{\mathrm{i}}\right)$ in the solution space.

Step-2 (Assign personal and global best position): For each particle of swarm, its current position is termed as personal best (pbest). The best value among all the current pbest values is named as global best value i.e. gbest.

Step-3 (Change in velocity and position of particles): Update the velocity $\left(\mathrm{v}_{\mathrm{i}}\right)$ and position $\left(\mathrm{x}_{\mathrm{i}}\right)$ of the particles of the swarm using the equations (4) and (5).

$$
\begin{aligned}
& v_{i}(t+1)=\omega v_{i}(t)+c_{1} r_{1}\left(P_{i}(t)-x_{i}(t)\right)+c_{2} r_{2}\left(G(t)-x_{i}(t)\right) \\
& x_{i}(t+1)=x_{i}(t)+v_{i}(t+1)
\end{aligned}
$$

where, $x_{i}=$ position of the $i^{\text {th }}$ particle 
$\omega=1.2$ (random weight used to balance the search algorithm)

$\mathrm{c}_{1}, \mathrm{c}_{2}=$ learning parameters where $\mathrm{c}_{1}, \mathrm{c}_{2}$ lies between 0 and 1

$\mathrm{r}_{1}, \mathrm{r}_{2}=$ random parameters between $[0,1]$

$\mathrm{P}_{\mathrm{i}}=$ best position found by the $\mathrm{i}^{\text {th }}$ particle

$\mathrm{G}=$ best position found by the population/ swarm

$\mathrm{t}=$ time index

Step-4 (Update personal and global best positions): The pbest (personal best) position of the particle is changed by comparing the value of new location with the previous location values. If the value of new location is better than the previous value then the value of pbest is updated with the new one; otherwise it will remain the same. Similarly the value of gbest is chosen by comparing the pbest values at that time. If the previous gbest value is better than the new value, it remains unchanged otherwise the value is updated.

Step- 5 (Stop Criterion): Repeat the above steps for all the particles of the swarm until the stopping criterion is satisfied. Here the stop criterion is the total number of iterations.

Step- 6 (Feature Selection): All the gbest values stored in the memory of particles, we obtained at the end, are considered as features selected by PSO algorithm. These features will now be used by ACO algorithm as its input i.e. as the population of ants in the beginning.

2. Recognizing the face using ACO

Before using ACO to recognize an image, we should assume that:

i. The image pixels are used to represent ants.

ii. Nodes are the features that have been selected.

Steps necessitate in recognizing an image with the help of ACO technique are:

Step- 1 (Initialization): Decide the population of the ants. The number of ants should be equal to the number of features we get from PSO. Assign the strength of the pheromone in between the nodes (based on the distance between them). Also find the number of nodes (number of iterations) to be travelled across.

Step- 2 (Generating the Ants): On each single feature, place an ant randomly. Each ant is randomly assigned to the feature and it must travel every node. An ant will move from one node to another with a probability, which can be calculated using equation (6).

$$
p_{i, j}=\frac{(\tau(i, j))(\eta(i, j))^{\beta}}{\sum(\tau(i, j))(\eta(i, j))^{\beta}}
$$

where $\tau(\mathrm{i}, \mathrm{j})$ is the strength of pheromone between edge $i$ and $j$

$\eta(i, j)=\frac{1}{d(i, j)}$ is called visibility and $\frac{1}{d(i, j)}$ is

distance between edge $\mathrm{i}$ and $\mathrm{j}$

$\beta=2$ (constant)

This value is stored in the memory of ant.

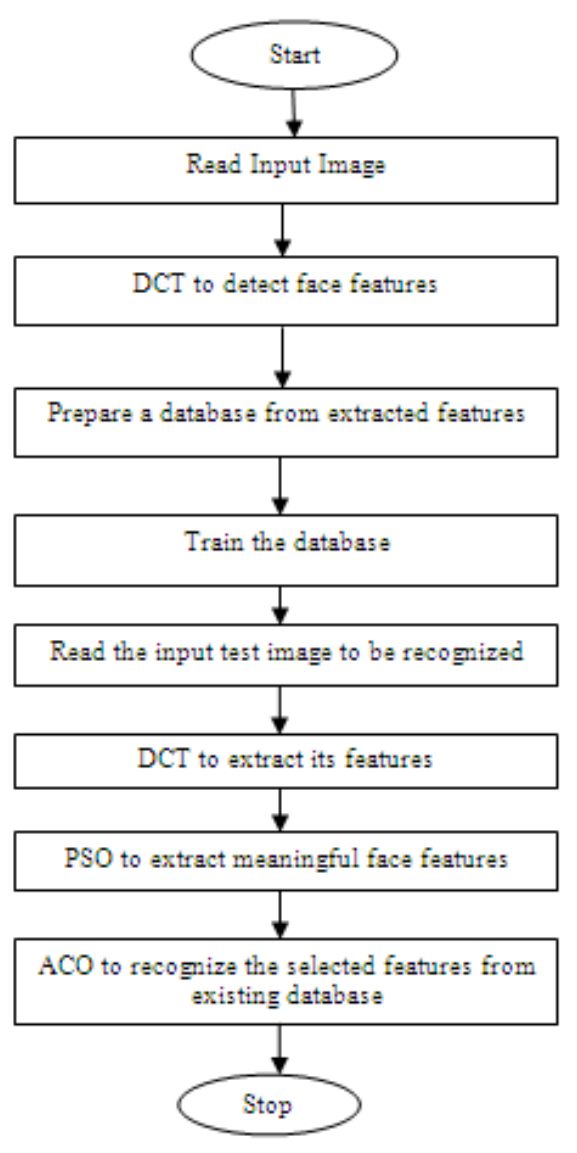

Fig. 2 Steps for feature extraction, selection and recognition in the hybrid algorithm

Step- 3 (Pheromone Updating): The two nodes which have the best probability value in between them is chosen as the next node to be travelled. The pheromone value is updated in between these nodes using the equations (7-9).

$\tau(i, j)=(1-\rho) \tau(i, j)+\square \tau_{i, j}$

where $\tau(i, j)$ is the value of pheromone on edge $\mathrm{i}$ and $\mathrm{j}$

$\rho$ is the rate of updating of pheromone between 0 and 1

$\square \tau_{i, j}$ is the amount of pheromone deposited typically given by

$\square \tau_{i, j}^{k}=\frac{1}{L_{k}}$ if ant k travels on edge $\mathrm{i}, \mathrm{j}$

$\square \tau_{i, j}^{k}=0 \quad$ if ant $\mathrm{k}$ travels on edge other than i,j (9) where $\mathrm{k}^{\text {th }}$ ant tour's length is represented by $\mathrm{L}_{\mathrm{k}}$

The node is marked as visited and path is marked as verified.

Step- 4 (Evaluation Criterion): In this step the evaluation criterion is the Euclidean distance among the features and the comparison of distances with the distances in the original image [4]. This can be calculated with the help of equation (10).

$d(p, q)=d(q, p)=\sqrt{\sum_{i=1}^{n}\left(q_{i}-p_{i}\right)^{2}}$ 
where $\mathrm{p}=\left(\mathrm{p}_{1}, \mathrm{p}_{2} \ldots \ldots, \mathrm{p}_{\mathrm{n})}\right.$ and $\mathrm{q}=\left(\mathrm{q}_{1}, \mathrm{q}_{2}, \ldots . \mathrm{q}_{\mathrm{n}}\right)$ are the points in Euclidean n-space. Also d is the distance between points $\mathrm{p}$ and $\mathrm{q}$.

Step- 5 (Checking the stop criterion): If all the features/nodes and paths have been visited by the ant (specified in Step- 1) then exit the algorithm otherwise continue.

Step- 6 (Result): The best value obtained in the end gives the output of the algorithm, which is the matched image in this case.

\section{RESULT AND DISCUSSION}

\section{Data Set}

In order to inspect the effectiveness of the algorithms, two images measuring $64 \times 64$ are stored in the database shown in Table 1 . Both of these images are matched with the test images, which are also stored in the database to compare the results.

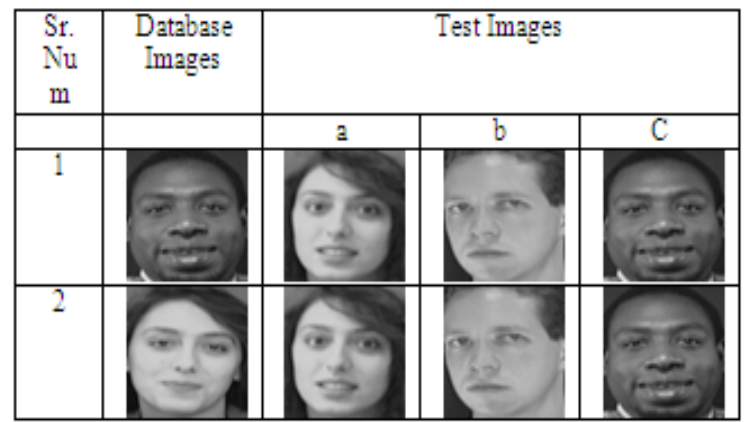

Table 1 Database and the Test Images

\section{Implementation and Discussion}

The results of proposed hybrid algorithm is matched with outcome of the simple optimization algorithms using the parameters Accuracy, Training Time, Recognition Time, Mean Square Error and Peak Signal to Noise Ratio. Experimental results of these are shown in the following graphs.

i. Accuracy

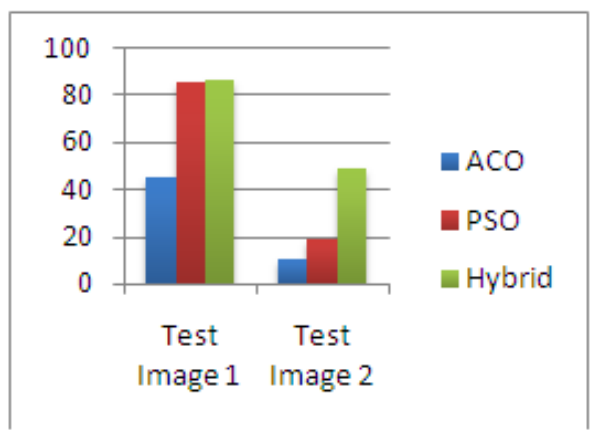

Fig. 3 Accuracy for test images using ACO, PSO and Hybrid approach

The graph above illustrates the accuracy of recognition of facial image using ACO, PSO and the Hybrid approach. It shows that the accuracy of recognition of PSO is better than ACO but less than the hybrid technique. ii. Training Time

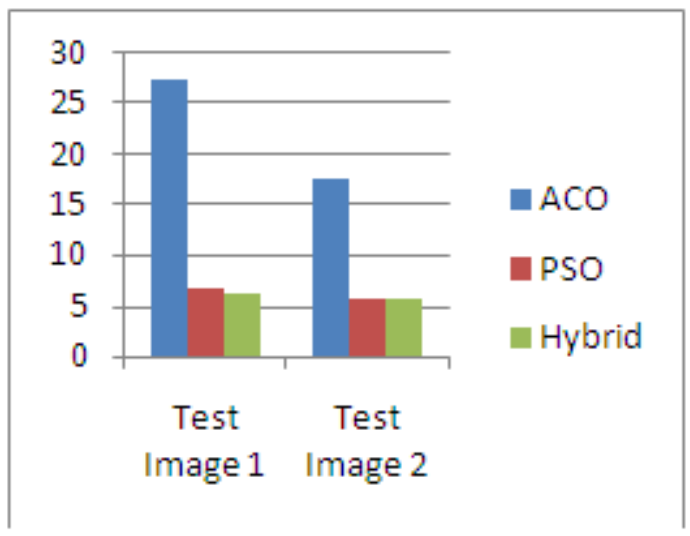

Fig. 4 Training Time for images using ACO, PSO and Hybrid approach

The graph above illustrates the training time required by images using ACO, PSO and the Hybrid approach. It shows that the time needed by ACO is much more than the time required by PSO and the hybrid technique.

iii. Recognition Time

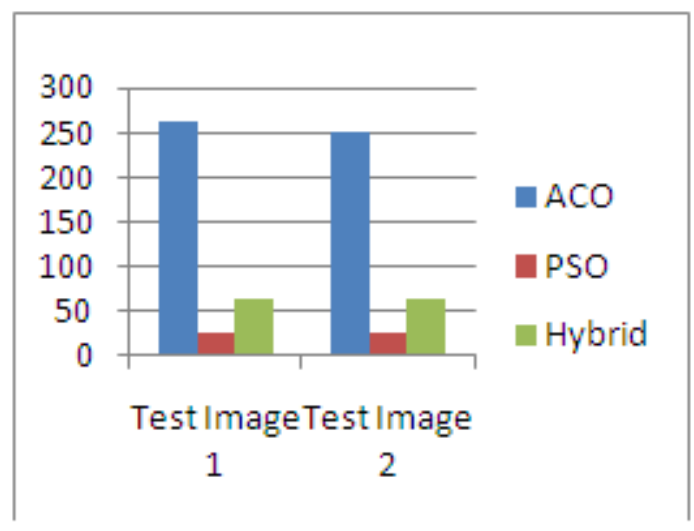

Fig. 5 Training Time for images using ACO, PSO and Hybrid approach

The above figure shows the time required by images using ACO, PSO and the Hybrid approach to recognize an image. It shows that the time needed by hybrid approach is less than ACO but more than the time required by PSO algorithm.

iv. Peak Signal to Noise Ratio

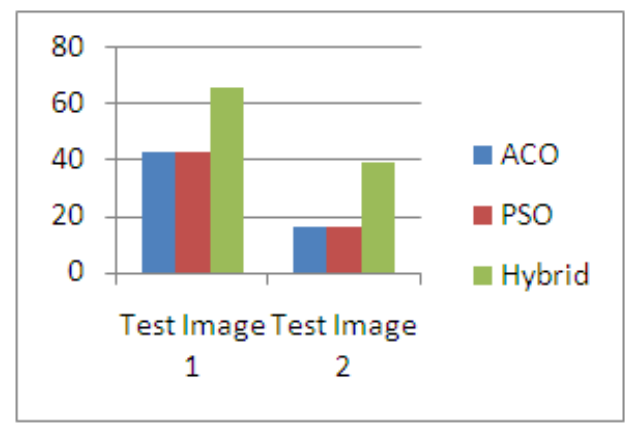

Fig. 6 PSNR values for images using ACO, PSO and Hybrid approach 
The above graph demonstrates the PSNR values of test images using ACO, PSO and the Hybrid approach to recognize an image. It shows that the highest PSNR value is achieved in case of hybrid approach than both the other techniques.

v. Mean Square Error

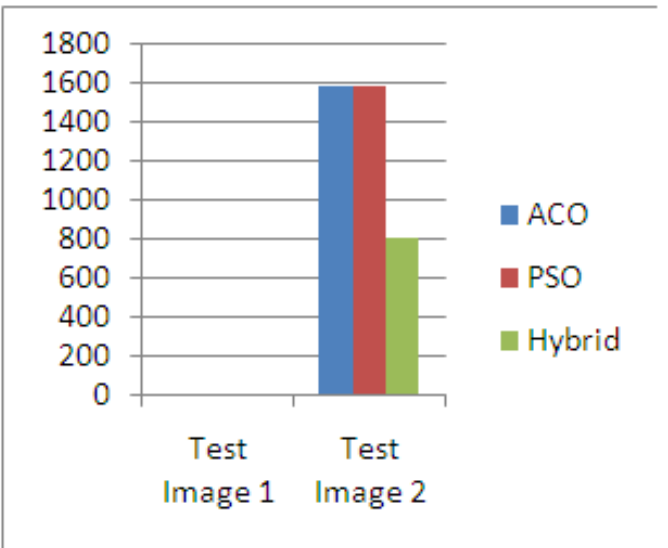

Fig. 7 MSE values for images using ACO, PSO and Hybrid approach

The above graph demonstrates the MSE values of test images using ACO, PSO and the Hybrid approach to recognize an image. In case of Test Image 1, the MSE value for ACO, PSO and Hybrid algorithm is 3.5, 3.5 and 1.7094 respectively; which is much smaller than the values of Test Image 2 . Hence it is not possible to show them here graphically. The graph shows that the lowest MSE value is achieved in case of hybrid approach than both the other techniques.

\section{CONCLUSION AND FUTURE SCOPE}

A new hybrid face recognition algorithm is propounded in this work. First of all the features are selected using the
Particle Swarm Optimization algorithm and then the features are recognized with the help of Ant Colony Optimization technique. Face recognition is done by comparing the distance between the features of the image obtained in both the cases. Experimental results show that the proposed hybrid algorithm has a better recognition rate than ACO and PSO algorithms. Also the PSNR and MSE values are greatest and least than the ACO and PSO algorithms respectively. In future the recognition time of hybrid algorithm can be decreased and accuracy can be increased by using more enhanced optimization techniques.

\section{REFERENCES}

[1] Darestani, M.R.Y., Sheikhan, M., and Kahademi, M. (2013). Face Recognition Using Conourlet- Based Features an Hybrid PSO- Neural Model. IEEE, 2013, pp. 181-186.

[2] Ramadan, R.M., and Kader, R.F.A. (2009). Particle Swarm Optimization for Human Face Recognition. IEEE. pp. 579- 584.

[3] Krisshnaa, N.L.A., Deepaka, V.K., Manikantana, K., and Ramachandran, S. (2014). Face recognition using transform domain feature extraction and PSO-based feature selection. Elsevier.

[4] Kaur, H., Panchal, V.K., and Kumar, R. (2013). A Novel Approach Based on Nature Inspired Intelligence for Face Feature Extraction and Recognition. IEEE, pp. 149-153.

[5] Ajith, A., He, G., and Hongbo, L. (2006). Swarm Intelligence: Foundations, Perspectives and Applications In Studies in Computational Intelligence.

[6] Kennedy, J. and Eberhart, R. (1995). Particle swarm optimization in Neural Networks. IEEE. Vol. 4, pp. 1942-1948.

[7] Dorigo, M., Birattari, M., and Stutzle, T. (2006). Ant Colony Optimization- Artificial ants as a Computational Intelligence Technique. IEEE Computational Intelligence Magazine. 